\title{
BMJ Open Development and feasibility of a guided and tailored internet-based cognitive- behavioural intervention for kidney donors and kidney donor candidates
}

\author{
Lieke Wirken, ${ }^{1,2}$ Henriët van Middendorp, ${ }^{1,2}$ Christina W Hooghof, ${ }^{3}$ \\ Tamara E Bremer, ${ }^{1,2}$ Sabine P F Hopman, ${ }^{3}$ Karlijn A M I van der Pant, ${ }^{4}$ \\ Andries J Hoitsma, ${ }^{3}$ Luuk B Hilbrands, ${ }^{3}$ Andrea W M Evers ${ }^{1,2}$
}

To cite: Wirken $L$, van Middendorp H, Hooghof CW, et al. Development and feasibility of a guided and tailored internet-based cognitive-behavioural intervention for kidney donors and kidney donor candidates. BMJ Open 2018;8:e020906. doi:10.1136/ bmjopen-2017-020906

- Prepublication history for this paper is available online. To view these files, please visit the journal online (http://dx.doi. org/10.1136/bmjopen-2017020906).

Received 5 December 2017

Revised 13 April 2018

Accepted 16 May 2018

Check for updates

${ }^{1}$ Institute of Psychology, Health, Medical and Neuropsychology Unit, Leiden University, Leiden, The Netherlands

${ }^{2}$ Department of Medical

Psychology, Radboud university medical center, Nijmegen, The Netherlands

${ }^{3}$ Department of Nephrology, Radboud university medical center, Nijmegen, The Netherlands

${ }^{4}$ Department of Internal Medicine/Nephrology, Renal Transplant Unit, Academic Medical Center, University of Amsterdam, Amsterdam, The Netherlands

Correspondence to

Lieke Wirken;

I.wirken@fsw.leidenuniv.n

\section{ABSTRACT}

Objectives Living donor kidney transplantation is currently the preferred treatment for patients with endstage renal disease. A subgroup of the kidney donor population experiences adjustment problems during or after the donation procedure (eg, anxiety or fatigue). There is a need for evidence-based interventions that decrease donation-related difficulties before or after donation. In the current study, a guided and tailored internet-based cognitive-behavioural therapy (ICBT) intervention for donors and donor candidates was developed and the feasibility and perceived effectiveness were evaluated.

Design Pilot study including qualitative and quantitative research methods for intervention development and evaluation.

Setting Living kidney donor population of two Dutch transplantation centres.

Participants Donors and healthcare professionals participated in focus group interviews conducted to identify intervention themes and to map attitudes towards internet-based interventions. In a pilot feasibility study, 99 donors and donor candidates participated, of whom 38 completed the screening. Eight donors or donor candidates with a risk profile (ie, impaired mental healthrelated quality of life $(\mathrm{HRQ} \mathrm{L})$ ) received and evaluated the intervention.

Interventions A guided and tailored ICBT intervention for donors and donor candidates was developed. Donation-related treatment modules, assignments and psychoeducation were integrated within an existing disease-generic ICBT intervention.

Outcome measures $\mathrm{HRQ} \mathrm{LL}$, anxiety and depression were assessed before and after the ICBT intervention. Additional questionnaires were included to identify specific problem areas of donor functioning to tailor the ICBT intervention to the donor's needs.

Results Different intervention themes were derived from the focus group interviews (eg, physical limitations, and donation-specific emotional and social-relational problems). Participants were satisfied about the intervention content $(7.7 \pm 0.8$ on a $0-10$ scale) and the therapeutic relationship ( $4.4 \pm 0.6$ on a $1-5$ scale), and indicated an improvement on domains of their treatment goals ( $3.2 \pm 0.7$ on a $1-4$ scale).
Strengths and limitations of this study

- This is the first study to develop an intervention for the subgroup of donors experiencing donation-related problems before or after donation.

- Both qualitative and quantitative research methods were applied for the development and evaluation of an internet-based cognitive-behavioural therapy intervention.

- Although with a small sample size, this pilot study provides promising indications for a useful and acceptable addition to psychosocial care for kidney donors.

Conclusion This study showed positive evaluations concerning both feasibility and perceived effectiveness of the tailored ICBT intervention in kidney donors and donor candidates, in line with previous studies using comparable ICBT treatment protocols in other populations. Future research should examine the possibilities of integrating the intervention into psychosocial care for kidney donors.

\section{INTRODUCTION}

Living donor kidney transplantation is the preferred treatment for patients with end-stage renal disease. This preference is based on higher patient and graft survival rates after transplantation than during dialysis treatment, and higher graft survival and generally shorter waiting times than in deceased donor kidney transplantation. ${ }^{1-3}$ Because of the increase in the number of living donor kidney transplantations during the past years, research on the optimisation of care of kidney donors has expanded. Living donors do not experience medical benefits from donation for themselves. Therefore, the protection of their well-being using evidencebased donor evaluation and treatment strategies to prevent and treat adverse consequences of donation is required. 
In order to apply for living kidney donation, donor candidates need to have a good physical and mental health-related quality of life (HRQoL). Previous research has indeed shown that the HRQoL of donors is generally equal to or above that of the general population before donation. Shortly after donation, donors experience a poorer HRQoL, which generally returns to baseline within 6-12 months postdonation (Wirken et al, 2018, submitted). ${ }^{45}$ However, it has also been shown that a proportion of donors (up to $35 \%$ ) develop adjustment problems during or after the donation procedure. For example, some donor candidates experience elevated levels of worrying about the upcoming surgery or the health status of the recipient, and some donors report high levels of fatigue or unmet expectations of the donation and transplantation outcomes. ${ }^{467}$

Within the current healthcare protocols for living kidney donors, psychosocial care before and after donation is not systematically embedded. Screening for donor eligibility is mainly based on medical criteria, and although the need for psychosocial screening is increasingly recognised, ${ }^{89}$ this is generally not conducted in an evidence-based manner, mainly because of the lack of large and encompassing prospective prediction studies in this population. Also, although there generally is a medical donor follow-up up to 12 months postdonation, both short-term (up to 6 months after donation) and longer term (eg, 12 months or longer after donation) psychosocial donor follow-ups are often lacking, even though recent guidelines recommend this. ${ }^{10}$ Reasons for this include a scarcity of large prospective studies showing predonation predictive factors of impaired HRQoL after donation. The few large prospective studies conducted have mainly shown worse predonation mental and physical functioning to be predictive of poorer HRQoL outcomes after donation, next to a number of individual risk and resilience factors. ${ }^{4}{ }^{11} 12$ Because of the differences in donor characteristics and the type of difficulties before or after donation, such as predonation worries or postdonation fatigue symptoms, tailored interventions that are specified to the individual characteristics, preferences and needs of donors and donor candidates to improve health or change behaviour could be useful. In this way the risk of longer-term adjustment problems in donor candidates could be reduced, and actual donors who experience donation-related problems could be supported.

Currently, only one psychosocial intervention is available for donor candidates who are scheduled for surgery, using predonation motivational interviewing in two telephonic sessions to reduce ambivalence towards donation. Results of the randomised controlled trial on the effectiveness of this intervention were positive, with a decline in ambivalence before donation in the intervention group, and better physical functioning, shorter recovery times, less anxiety and fewer unexpected donation-related family problems 3 months after donation. ${ }^{13}$ To our knowledge, no empirically supported psychosocial interventions for donors and donor candidates with elevated distress levels for a variety of donation-related difficulties are available. Therefore, there is a need for evidence-based interventions that focus on (1) decreasing difficulties in donor candidates during the donor screening procedure or (2) reducing negative consequences after donation. ${ }^{145}$

Cognitive-behavioural therapy is an evidence-based psychological intervention which is used worldwide to treat mental health conditions, focusing on the replacement of maladaptive cognitions and coping strategies by more positive cognitions and behaviours. To this aim, different techniques are used, including the training of problem-solving skills, relaxation training, stress management, and cognitive restructuring which entails changing unhelpful cognitions into helpful ones. ${ }^{16} 17$ Previous research showed that cognitive-behavioural therapy that is provided via the internet (internet-based cognitive-behavioural therapy or ICBT) could be as effective as faceto-face treatments in the improvement of physical and psychological functioning in patients with mental and physical health problems. ${ }^{18-20}$ One component that has found to be associated with the effectiveness of ICBT is online guidance by a therapist during the treatment, for example by means of email messages providing motivating remarks and feedback on assignments. ${ }^{21-23}$ Additionally, there is increasing evidence that the tailoring of interventions to the specific risk and resilience factors of individuals increases the effectiveness of (I) CBT. ${ }^{24-26}$ The advantages of cognitive-behavioural interventions using the internet in comparison with face-to-face treatment in the hospital include that individuals can follow the treatment from their own homes, which improves the application of learnt techniques directly in their own environment, and that treatment can be followed in their own time, improving flexibility and preventing travel costs and waiting times. ${ }^{27}$

In conclusion, current psychosocial care for living kidney donors and donor candidates at risk for long-term adjustment problems is limited and not evidence-based. Guided and tailored ICBT could be a valuable addition to current psychosocial care for living donors, in providing predonation support or short-term or longer-term psychosocial follow-up for donors with a risk profile. The current study describes the development of a guided and tailored ICBT intervention for donors and donor candidates, and examines the satisfaction with and feasibility of this intervention in a pilot study in a small group of donors and donor candidates.

\section{MATERIALS AND METHODS}

Three steps have been taken to develop the guided and tailored ICBT intervention for kidney donors and donor candidates and test its feasibility.

\section{Focus group interviews}

Three focus group interviews were conducted with living kidney donors from two transplantation centres from 
different parts of the Netherlands, in order to provide a good representation of the Dutch donor population in terms of sociodemographic characteristics and cultural background (Radboudumc Nijmegen and Academic Medical Center Amsterdam). Also, one focus group interview with transplant professionals was conducted (nephrologists, nurses and medical social workers) in one transplantation centre (Radboudumc). After these focus group interviews, saturation was reached. The aim of these focus groups was to identify possible themes that would be considered relevant by donors and transplant professionals to be included in a donor-specific intervention, and to map general attitudes towards internet-based interventions. A health psychologist with background experience in group therapy guided all focus group interviews as a mediator and another psychologist was present as an observer. Focus group interviews were audio-recorded and video-recorded. Participants (donors as well as transplant professionals) signed informed consent at the start of the interviews.

The questioning route was developed based on donation consequences from previous research and clinical practice, and was evaluated in a team of experts involved in living donation research and practice. The focus group interviews started with questions about the possible physical limitations and psychological consequences before and following donation, the influence of the donation on social relationships and daily functioning, and the ways by which donors handled potential problems in their functioning. Also, questions were asked about the perceived potential need for additional psychosocial care and the themes on which this care should be focused according to both donors and transplant professionals. Further, the attitudes of both groups towards ICBT interventions were explored.

\section{Evaluation and adjustment of existing ICBT interventions for patients with somatic conditions}

Before the start of the current study, our research group developed a generic, guided and tailored ICBT intervention, based on evidence-based face-to-face CBT for patients with chronic somatic conditions, which was aimed at all patients showing adjustment problems at a certain point in their disease trajectory. The content of the intervention was not primarily focused on chronic distress or chronic physical symptoms, but consists of general cognitive-behavioural principles to help people deal with a variety of adjustment problems of different durations. For example, assignments and psychoeducational texts in the intervention focus on coping with fatigue or temporary physical limitations or excessive worrying, which could be particularly helpful in this donor population. The effectiveness of this ICBT intervention was demonstrated in a randomised controlled trial in different patient populations. ${ }^{26}{ }^{28}$ Based on the themes identified in the focus group study and previous (intervention) studies in living kidney donors, the existing generic ICBT intervention was adjusted to incorporate specific donation-related treatment modules, exercises and psychoeducation, resulting in the ICBT intervention for donors. This ICBT intervention is a therapist-guided intervention tailored to the individual treatment goals of donors.

\section{Feasibility testing of the ICBT intervention}

Research procedure feasibility study

Due to the possibility to tailor the ICBT intervention to the specific needs of the donor or donor candidate, both groups could participate in the intervention. Therefore, individuals who donated a kidney for transplantation or who were registered as donor candidates from November 2014 to August 2015 were invited to participate in the study by means of an information letter. When donors or donor candidates were interested in participating in the study, a two-step procedure was followed. After signing informed consent, they were asked to fill in several questionnaires to provide a risk categorisation based on their current mental HRQoL or psychological distress (see next paragraph). Participants with elevated distress levels were invited to participate in the pilot intervention study, in which they received the ICBT intervention before or after donation. The intervention was conducted with the understanding that participants appropriately met the kidney donation candidacy criteria. After finishing the intervention, participants received a questionnaire to evaluate the content and procedure of the ICBT intervention. Evaluation measures were based on the main areas of limitations that kidney donors report during or after the donation procedure, as have been reported in a previous systematic review and meta-analysis of the literature. ${ }^{4}$ Next to these subjective evaluations, the intervention was evaluated more objectively with technical data on the use of the intervention and the intervention duration (eg, the frequency of logins per donor or donor candidate).

\section{Screening questionnaires}

Exploring donor candidacy criteria was outside the scope of this study. In the limited amount of previous prospective research in kidney donors, impaired baseline physical and psychosocial functioning were the most consistent risk factors for poorer HRQoL after donation. ${ }^{4}$ Because of the psychological focus of the intervention, donors and donor candidates were, therefore, screened on psychosocial functioning to identify donors who could benefit from the ICBT intervention, based on current mental HRQoL or psychological distress, assessed with the RAND Short Form-36 Health Status Inventory - mental health subscale $\left(\right.$ RAND SF-36) ${ }^{29}$ and the Hospital Anxiety and Depression Scale (HADS).$^{30} \mathrm{~A}$ psychological risk profile was defined as RAND SF-36 mental health subscale scores $\leq 48$ or HADS anxiety or depression scores $\geq 5$.

The RAND SF-36 is a 36-item questionnaire assessing eight HRQoL dimensions. Four dimensions measure psychological functioning: emotional well-being, role limitations due to emotional problems, social functioning and energy. These are summarised in the Mental Health 
Composite Score, which was used in the screening. Higher scores represent higher mental HRQoL. ${ }^{29}$

The HADS consists of seven items measuring anxiety (eg, 'I get a sort of frightened feeling as if something awful is about to happen') as well as seven items on depressive symptoms (eg, 'I feel as if I am slowed down'). Scores are rated on a 4-point scale; higher scores represent more anxiety or depressive symptoms. ${ }^{30}$

\section{Intervention-tailoring questionnaires}

To tailor the ICBT intervention to individual treatment goals, additional questionnaires were used to identify specific problem areas of functioning, distinguishing physical functioning (RAND SF-36), ${ }^{29}$ fatigue (Checklist Individual Strength-Fatigue Scale (CIS) short version) ${ }^{31}$, neuroticism (NEO Personality Inventory Revised-Neuroticism) ${ }^{32}$ and social-relational functioning (Interpersonal Sensitivity Measure ${ }^{33}$ and the Inventory for Social Reliance $\left.^{34}\right)$.

\section{ICBT intervention evaluation measures}

\section{Satisfaction with the ICBT intervention}

The information provision before the intervention, the degree to which expectations of the intervention were met, the general satisfaction with the intervention, the donor beliefs about a sustainable effect of the intervention and the extent to which donors would recommend the intervention to relatives were evaluated directly after finishing the intervention. Furthermore, the dedication towards the intervention and the effect of the intervention on treatment goals were evaluated. All evaluations were made on a 4-point scale ( $1=$ no to $4=$ certainly). Higher scores represent more satisfaction with the intervention, more active participation in the intervention and more effect of the intervention on treatment goals.

\section{Feasibility of the ICBT intervention}

The comprehensibility and usefulness of the assignments and psychoeducational texts were assessed using a 7-point scale $(0=$ very poor to $6=$ very good $)$.

\section{Therapeutic relationship}

The therapeutic relationship during the ICBT intervention was assessed using the Working Alliance Inventory (WAI $^{35} 36$ and the Internet-Specific Therapeutic Relationship Questionnaire (ITRQ). ${ }^{37}$

The WAI assesses the working alliance concepts of therapeutic relationships, consisting of three subscales, namely agreement on therapy goals, agreement on therapy tasks and the development of a strong relational bond between the patient and the therapist, assessed on a $1-5$ scale $(1=$ never to 5 =always $){ }^{35} 36$

The ITRQ measures internet-specific aspects of the therapeutic relationship during internet-based interventions, consisting of two 4-item subscales: internet-specific time and attention (which assesses time lag aspects of communication and receiving sufficient attention from the ICBT therapist), and internet-specific reflection and comfort (which comprises the sharing of information with the therapist and home as the treatment environment), on a $1-10$ scale ( $1=$ totally disagree to $10=$ totally agree). ${ }^{37}$

\section{Usage of the ICBT intervention}

To gain insight into the use of the intervention website with objective parameters, the frequency of logins per donor and the total intervention duration were assessed.

\section{Patient and public involvement}

The study was initiated based on the clinical question from healthcare professionals on how to identify and support donors at risk for impaired functioning after donation, which was sometimes observed in clinical practice, but for whom no protocol was available. The research questions and outcome measures were thus based on the clinical expertise of the healthcare professionals who are specialised in donor care, including coordinating nurses and medical social workers. To provide input for relevant themes of the ICBT intervention and to assess the feasibility thereof, focus groups with (potential) donors and healthcare professionals involved in donor care were held. Furthermore, donors were involved as research participants in the pilot intervention. The study results have been and will be disseminated to the donors and healthcare professionals involved in donor care by means of presentations at patient associations and professional conferences.

\section{Statistical analyses}

Focus group interviews were analysed using content analysis to deduce themes for the ICBT intervention. ${ }^{38}$

\section{Changes in HRQoL before and after the ICBT intervention}

Because of the small sample size, no formal statistics were performed on changes in functioning. Instead, descriptive statistics and effect sizes for small samples (Hedges' g) were calculated for changes in HRQoL (physical and psychosocial functioning) before and after the ICBT intervention, using the RAND SF-36, HADS and CIS short version. ${ }^{29-31}$ An effect size of 0.2 represents a small effect, 0.5 a medium effect and 0.8 a large effect. ${ }^{39}$

\section{Satisfaction with the ICBT intervention}

Descriptive statistics were performed on the separate items on satisfaction with the intervention (4-point scale).

\section{RESULTS}

\section{Intervention development}

Focus group interviews

\section{Participants}

Focus group interviews were conducted with 13 donors postdonation, of whom $69 \%$ were female and who had a mean age of 58.8 years (age range $30-74$ years). Most donors had donated directly to the person they knew $(77 \%)$, but also kidney donor exchange $(8 \%)$ and altruistic $(15 \%)$ donors were represented. In the focus group interview with transplant professionals, one nephrologist, 
Table 1 Demographic characteristics of donors and transplant professionals participating in the focus group interviews and pilot study of the internet-based cognitivebehavioural therapy (ICBT) intervention

Mean \pm SD (range)

n (\%)

\begin{tabular}{|c|c|}
\hline \multicolumn{2}{|c|}{ Participants of the focus group interviews } \\
\hline \multicolumn{2}{|l|}{ Donor characteristics $(n=13)$} \\
\hline Female gender & $9(69)$ \\
\hline Age (years) & $58.8 \pm 11.5(30-74)$ \\
\hline \multicolumn{2}{|l|}{ Donation type } \\
\hline Direct & $10(77)$ \\
\hline Kidney exchange & $1(8)$ \\
\hline Altruistic & $2(15)$ \\
\hline Time since donation (years) & $2.4 \pm 1.7(0.6-7.0)$ \\
\hline \multicolumn{2}{|c|}{ Donation professionals' characteristics $(n=5)$} \\
\hline \multicolumn{2}{|l|}{ Type professional } \\
\hline Nephrologist & $1(20)$ \\
\hline Donation coordinating nurse & $1(20)$ \\
\hline Medical social worker & $3(60)$ \\
\hline $\begin{array}{l}\text { Work experience in transplantation } \\
\text { care (years) }\end{array}$ & $6.0 \pm 4.2(3-13)$ \\
\hline
\end{tabular}

Participants of pilot study on the ICBT intervention Characteristics of donors and donor candidates $(n=8)$

\begin{tabular}{|c|c|}
\hline Female gender & $5(63)$ \\
\hline Age (years) & $58.6 \pm 11.4(38-74)$ \\
\hline \multicolumn{2}{|l|}{ Donation type } \\
\hline Direct & $6(75)$ \\
\hline Kidney exchange & $1(13)$ \\
\hline Altruistic & $1(13)$ \\
\hline \multicolumn{2}{|c|}{ Time between donation and ICBT intervention (months) } \\
\hline Before donation $(n=2)$ & $M=1.5 \pm 0.4$ \\
\hline Time after donation $(n=6)$ & $M=6.4 \pm 3.6$ \\
\hline
\end{tabular}

one coordinating nurse and three medical social workers participated, with a mean work experience in the field of transplantation of 6.0 years (range 3-13 years) (see table 1 for participants' characteristics).

\section{Intervention themes}

In the focus group interviews, donors and transplant professionals identified a number of themes that were considered to be useful to include in donor interventions. These included high levels of fatigue and related concentration problems, pain, problems with getting back to work, dealing with the simultaneous presence of the donor's own temporary limitations after surgery and limitations for the recipients, worries about the recipient (eg, graft failure or rehospitalisation), dealing with waiting times for transplantation after being eligible for donation, the influence of donation on family life and relationships, and sharing donation experiences with other donors or donor candidates.
These themes were used for the adjustment of the existing ICBT intervention. Finally, despite overall satisfaction with the consultations with medical social workers during eligibility screening, donors indicated a need to optimise the follow-up contacts after donation, with more attention for psychosocial issues.

\section{Feasibility testing of the ICBT intervention}

\section{Participants of the pilot study}

Ninety-nine individuals (47 donor candidates and 52 donors) were invited to participate in the pilot study. Of these, 14 donor candidates (30\% response rate) and 24 donors (46\% response rate) completed the screening questionnaire, of whom 16 donors (42\%) had a higher risk profile. Three of these donors indicated that they experienced only minor difficulties and were not interested in the intervention, and five donors did not respond to the invitation for intervention. Two donor candidates and six donors received the tailored ICBT intervention to reduce donation-related distress, of whom five were female and three were male. Age varied from 38 to 74 years, with a mean age of $59 \pm 11$ years. Of donor candidates, one was on the kidney exchange programme and one intended to donate directly to the person he/she knew; of the donors, five donated directly to the person they knew and one donated anonymously (table 1).

\section{Procedure of the ICBT intervention}

After signing informed consent, participants were invited for a face-to-face appointment with their therapist to get acquainted and formulate treatment goals based on the screening questionnaire or current causes of distress. The intervention was provided by one therapist with a master's degree on clinical psychology and a clinical registration as health psychologist. The intervention consisted of five different treatment modules with focus on the following items: physical limitations, fatigue, negative mood, social functioning and donation-related problems in daily life (see figure 1). After the face-to-face consultation with their therapist, an instruction on the use of the website was provided by telephone by a research assistant. Afterwards, the donor (candidate) could log on to the secure website to start with the ICBT intervention. The ICBT website contains an integrated platform in which donors can find informative reading texts and personal assignments (assigned by the therapist), do exercises (eg, relaxation), and send and read messages (including homework and feedback) to and from the therapist. Participants followed one or two tailored treatment modules based on their treatment goals. Tailored assignments and psychoeducational texts based on cognitive-behavioural techniques related to these individual treatment goals were placed on the website by the therapist and completed online by the donor. The therapist provided personalised feedback on the exercises weekly via a message box, and donors could reply to the therapist or ask questions via this message box. After finishing the personalised modules, the treatment was ended with a final treatment module about relapse prevention and the 


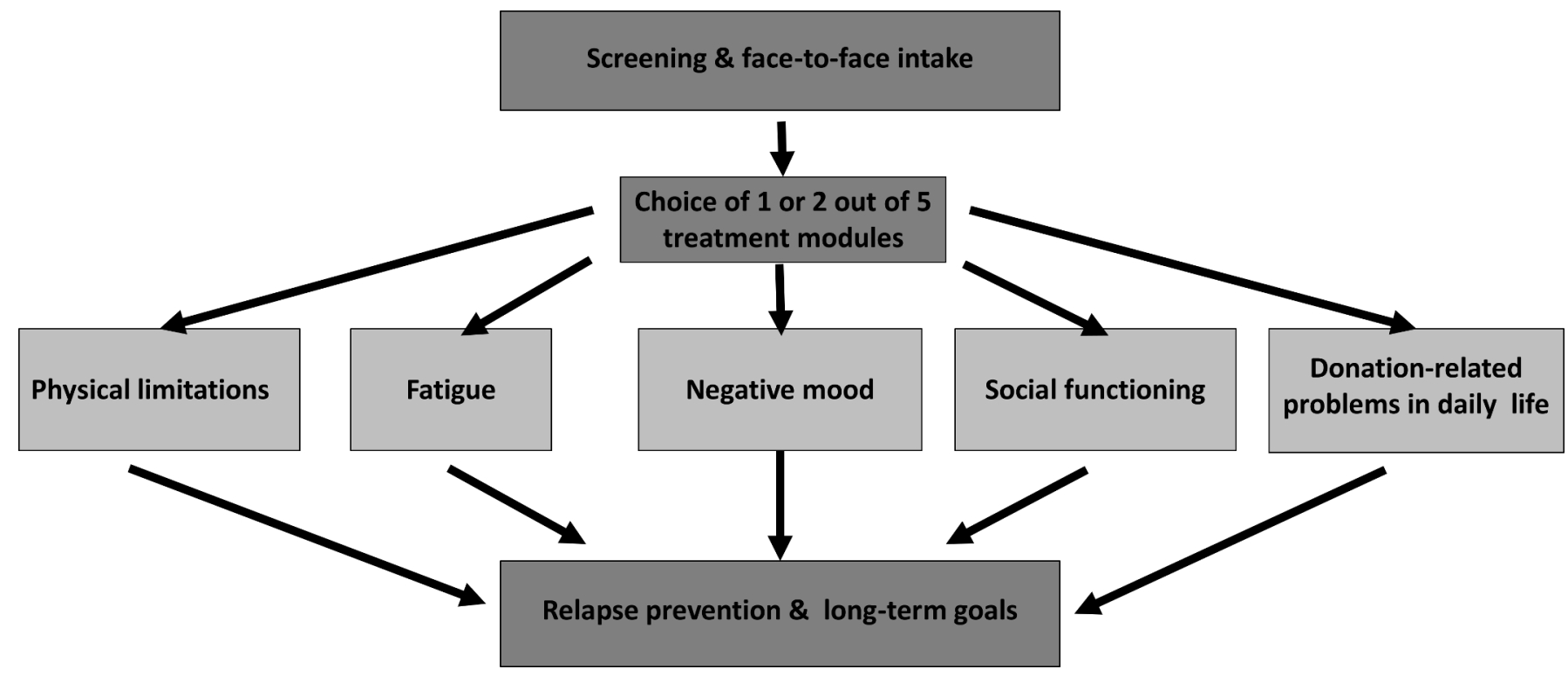

Figure 1 Intervention procedure.

formulation of long-term goals (see figure 1 for the structure of the intervention).

Within the group of eight participants, all five treatment modules were applied at least once; two donors worked on negative mood and donation-related problems in daily life, two donors on negative mood and fatigue, one donor on physical limitations and social functioning, one donor on fatigue and social functioning, one donor on negative mood, and one donor on fatigue. The intervention had a mean duration of $11.6 \pm 5.3$ weeks (range 3-18 weeks). Two donor candidates followed the intervention before donation with a short time until donation of 1.2 and 1.8 months, respectively. Six donors followed the intervention after donation, with a mean time between donation and intervention of $M=6.4 \pm 3.6$ months (range $2-10$ months).

\section{Evaluation of the intervention}

Descriptive statistics and effect sizes showed preliminary small-sized to medium-sized improvements from before to after the ICBT intervention in several aspects of mental HRQoL, with the largest improvements in the mental component score and energy subscale of the RAND SF-36 (Hedges' $g>0.50$ ), and small improvements in some aspects of physical HRQoL, including pain and general health perceptions (Hedges' $\mathrm{g}>0.20$ ) (table 2).

Participants were satisfied with the ICBT intervention $(M=7.7$, range $6-8$ on a $0-10$ scale). On a $1-4$ scale they reported that the intervention met their expectations $(M=3.4)$, that they had received appropriate information before the start of the treatment $(M=4.0)$ and that they thought that the treatment effect would persist in the longer term $(M=3.4)$. Most donors would recommend the ICBT intervention to future donors $(M=3.6)$. With regard to the dedication to the treatment, participants reported that they usually conducted the assignments that the therapist sent them $(M=3.0)$ and that they worked on the treatment goals intensively $(M=3.3)$, practising the assignments and integrating the learnt strategies in daily life $(M=3.1)$. Most participants did not have a fixed moment of time to conduct the assignments $(M=2.0)$. Participants reported a perceived effectiveness of the intervention, by indicating an improvement on the domains of their treatment goals $(M=3.2)$. On a scale from 0 to 6 , donors reported that the assignments were easily understandable $(M=5.1)$, had an appropriate length $(M=4.4)$ and were considered useful $(M=4.4)$. The user-friendliness was evaluated with a 7.0 on a 10-point scale (table 3 ).

The therapeutic relationship during the ICBT intervention was evaluated very positively, with a high agreement on therapy goals $(M=4.2)$ and on therapy tasks $(M=3.8)$, and the development of a strong relational bond between the patient and the therapist $(M=4.4)$, on a $1-5$ scale. Also, internet-specific aspects of the therapeutic relationship were rated very positively, such as the ITRQ subscale 'Internet-specific time and attention' which describes the time lag aspects of communication and receiving sufficient attention from the ICBT therapist $(M=8.9)$, and the subscale 'Internet-specific reflection and comfort' which includes the sharing of information with the therapist and the home as the treatment environment $(M=7.8)$, on a $1-10$ scale (table 3).

\section{Usage of the ICBT intervention}

Concerning the usage of the ICBT intervention by donors, the mean number of logins per donor was $M=29.1$ (range 3-55), with a duration of $M=9.4 \mathrm{~min}$ per login (range 0.5-128). The number of assignments that was completed by donors was $M=22.9$ (range $5-36$ ), and the mean number of messages they sent to their therapist was $M=13.6$ (range 1-24). The mean total intervention duration was $M=7.1$ hours (range $0.7-19.7$ hours).

With regard to the usage of the website by the psychologist who guided the intervention, the mean time duration of one login of the therapist was $M=47.0 \mathrm{~min}$. The 
Table 2 Pre-ICBT and post-ICBT intervention measures for eight donors or donor candidates

\begin{tabular}{|c|c|c|c|c|c|c|}
\hline \multirow[b]{2}{*}{ Outcome measures } & \multicolumn{2}{|c|}{ Before ICBT } & \multicolumn{2}{|c|}{ After ICBT } & \multirow{2}{*}{$\begin{array}{l}r \text { (pre-ICBT and } \\
\text { post-ICBT) }\end{array}$} & \multirow{2}{*}{$\begin{array}{l}\text { Effect size } \\
\text { (Hedges' g) }\end{array}$} \\
\hline & $M$ & SD & $M$ & SD & & \\
\hline \multicolumn{7}{|c|}{ Health-related quality of life (RAND SF-36) $\dagger$} \\
\hline \multicolumn{7}{|l|}{ Physical HRQoL } \\
\hline Physical Health Composite Score & 54.13 & 4.7 & 56.38 & 4.1 & 0.82 & 0.30 \\
\hline Physical functioning & 55.50 & 4.6 & 55.38 & 3.1 & 0.96 & -0.01 \\
\hline $\begin{array}{l}\text { Role limitations-physical health } \\
\text { problems }\end{array}$ & 54.13 & 5.3 & 55.00 & 8.9 & -0.14 & 0.15 \\
\hline Pain & 55.63 & 6.6 & 57.75 & 8.1 & 0.25 & 0.35 \\
\hline General health perceptions & 51.00 & 5.2 & 55.00 & 3.9 & 0.94 & 0.30 \\
\hline \multicolumn{7}{|l|}{ Psychological HRQoL } \\
\hline Mental Health Composite Score & 51.25 & 6.9 & 54.25 & 2.8 & 0.24 & 0.61 \\
\hline Emotional well-being & 48.88 & 7.3 & 50.88 & 5.9 & 0.31 & 0.35 \\
\hline $\begin{array}{l}\text { Role limitations - mental health } \\
\text { problems }\end{array}$ & 49.75 & 8.5 & 52.63 & 8.3 & -0.20 & 0.43 \\
\hline Social functioning & 54.63 & 4.8 & 53.00 & 4.2 & 0.40 & -0.39 \\
\hline Energy & 51.25 & 6.6 & 57.50 & 7.0 & 0.38 & 1.02 \\
\hline \multicolumn{7}{|c|}{ Anxiety and depression (Hospital Anxiety and Depression Scale)‡ } \\
\hline Depression & 2.00 & 1.9 & 1.88 & 1.6 & 0.63 & 0.06 \\
\hline Anxiety & 4.00 & 2.7 & 4.50 & 3.0 & 0.05 & -0.24 \\
\hline \multicolumn{7}{|l|}{ Fatigue (CIS short version)‡ } \\
\hline Fatigue & 9.75 & 6.4 & 9.63 & 6.0 & 0.93 & 0.01 \\
\hline
\end{tabular}

$\mathrm{n}=8$.

${ }^{\star}$ Effect sizes were presented using negative values indicating a reduction of symptoms.

†Higher scores represent better functioning.

‡Higher scores represent more symptoms.

CIS, Checklist Individual Strength-Fatigue Scale; HRQoL, health-related quality of life; ICBT, internet-based cognitive-behavioural therapy; RAND SF-36, RAND Short Form-36 Health Status Inventory.

therapist sent an average of 15 (range 3-25) messages per donor.

\section{DISCUSSION}

In the present study, a therapist-guided internet-based CBT intervention was developed, tailored to the specific needs of kidney donors or donor candidates. The feasibility and perceived effectiveness of the intervention were evaluated in a small group of (potential) kidney donors, showing positive evaluations with regard to HRQoL changes, satisfaction with the intervention, perceived effectiveness, user-friendliness and therapeutic relationship.

An existing tailored ICBT intervention that has been found effective in patients with different somatic conditions ${ }^{2628}$ was slightly adapted to the specific characteristics of the donor population, based on focus group interviews. Relevant themes deduced for the content of the intervention included physical difficulties, work resumption problems, and emotional and social-relational problems. Accordingly, donor-specific treatment modules, assignments and psychoeducational texts on the themes that were derived from the focus group interviews were included in the ICBT intervention. The attitude towards an internet-based intervention for kidney donors and donor candidates was mainly positive. After a first short screening on elevated levels of psychological distress, a small, but representative group of kidney donors or kidney donor candidates with a high-risk profile followed and evaluated the ICBT intervention. Although the small sample size prevented formal statistics, preliminary indications were found for improvement of some, mainly mental HRQoL aspects of participants after the ICBT intervention in comparison with baseline assessments. Furthermore, participants were satisfied about the information provision, the content and the usefulness of the ICBT intervention, and were very positive about the possibility to build a strong relationship with their therapist. Also, the user-friendliness was evaluated positively and participants indicated an improvement on the domains of their treatment goals. Thus, this pilot study provides promising indications for a useful and acceptable potential addition to psychosocial care for kidney donors.

The use of web-based interventions in healthcare has been increasing during the last decades. For kidney donors and donor candidates, web-based interventions could be 
Table 3 Results of the pilot study of the ICBT intervention

Mean \pm SD

(range)

\section{Generic satisfaction with the ICBT intervention}

I am satisfied with the ICBT intervention. (0-10) $7.7 \pm 0.8(6-8)$

The intervention met my expectations. (1-4) $3.4 \pm 0.7(2-4)$

I received appropriate information before the $4.0 \pm 0.0$ (4-4)

start of the intervention. (1-4)

I have faith that the treatment effect would $\quad 3.4 \pm 0.9(2-4)$ persist in the longer term. (1-4)

I would recommend the ICBT intervention to $3.4 \pm 1.1(1-4)$ relatives who consider to donate a kidney.

$(1-4)$

\section{Perceived effectiveness of the intervention}

Due to the intervention, my functioning on the $3.2 \pm 0.7(2-4)$

domains of my treatment goals improved. (1-4)

\begin{tabular}{|c|c|}
\hline \multicolumn{2}{|l|}{ Dedication to the intervention } \\
\hline $\begin{array}{l}\text { During the intervention, I conducted all the } \\
\text { assignments completely. (1-4) }\end{array}$ & $3.0 \pm 1.1(1-4)$ \\
\hline $\begin{array}{l}\text { I worked very intensively on my treatment } \\
\text { goals. }(1-4)\end{array}$ & $3.3 \pm 0.7(2-4)$ \\
\hline $\begin{array}{l}\text { I practised and integrated the learnt strategies } \\
\text { into daily life. }(1-4)\end{array}$ & $3.1 \pm 0.4(2-4)$ \\
\hline $\begin{array}{l}\text { I made time for the intervention at a fixed } \\
\text { moment on the day. }(1-4)\end{array}$ & $2.1 \pm 0.6(1-3)$ \\
\hline $\begin{array}{l}\text { The intervention contributed to an } \\
\text { improvement on dealing with problems related } \\
\text { to the domains of my treatment goals. (1-4) }\end{array}$ & $3.1 \pm 0.6(3-4)$ \\
\hline $\begin{array}{l}\text { I think the assignments were generally easily } \\
\text { understandable. (0-6) }\end{array}$ & $5.1 \pm 1.4(2-6)$ \\
\hline $\begin{array}{l}\text { I think the assignments had an appropriate } \\
\text { length. }(0-6)\end{array}$ & $4.3 \pm 1.0(3-6)$ \\
\hline I think the assignments were useful. (0-6) & $4.4 \pm 1.1(3-6)$ \\
\hline $\begin{array}{l}\text { Which grade would you give to the user- } \\
\text { friendliness of the website? }(1-10)\end{array}$ & $7.0 \pm 1.4(4-8)$ \\
\hline \multicolumn{2}{|l|}{ The therapeutic relationship } \\
\hline \multicolumn{2}{|l|}{ Working alliance (WAl) } \\
\hline Agreement on therapy goals. $(1-5)$ & $4.2 \pm 0.6(3.0-4.8)$ \\
\hline Agreement on therapy tasks. (1-5) & $3.8 \pm 1.0(1.8-4.8)$ \\
\hline $\begin{array}{l}\text { Relational bond between the patient and } \\
\text { the therapist. (1-5) }\end{array}$ & $4.4 \pm 0.6(3.5-5.0)$ \\
\hline \multicolumn{2}{|c|}{ Internet-specific aspects of the therapeutic relationship (ITRQ) } \\
\hline $\begin{array}{l}\text { The time lag aspects of communication and } \\
\text { receiving sufficient attention from the ICBT } \\
\text { therapist. (1-10) }\end{array}$ & $\begin{array}{l}8.9 \pm 1.1(7.5- \\
10.0)\end{array}$ \\
\hline $\begin{array}{l}\text { Sharing of information with the therapist and } \\
\text { the home as the treatment environment. (1-10) }\end{array}$ & $\begin{array}{l}7.8 \pm 2.2(5.0- \\
10.0)\end{array}$ \\
\hline
\end{tabular}

ICBT, internet-based cognitive-behavioural therapy; ITRQ, InternetSpecific Therapeutic Relationship Questionnaire; WAI, Working Alliance Inventory.

very suitable by saving time and costs related to travelling towards the transplantation centre. The number of living donor kidney transplantations is increasing, which means that the number of donors that could benefit from an intervention will be growing in the upcoming years. Also, the intervention for kidney donors could be generalised to other living donor populations in the future, such as living liver donors.

The positive evaluation of the ICBT intervention could be a result of the tailored intervention approach, which has the advantage of matching the intervention to the needs and characteristics of that specific donor. Both the focus groups interviews in the current study as well as previous literature indicated that most kidney donors experience no or a limited degree of problems before or after donation. However, a subgroup of kidney donors experience difficulties, and these are mostly very specific for the situation of that donor. Therefore, it is hard to develop a generic intervention protocol that could be used for all donors. With the tailoring approach, each kidney donor at risk can receive the optimal intervention. Also, the very positive evaluation of the contact with the therapist could have contributed to the positive evaluation of the guided ICBT intervention by kidney donors and donor candidates.

Due to the novelty of the internet-based approach of donor coaching, the current study was conducted in a small group of kidney donors and donor candidates, to test the satisfaction and feasibility of the newly developed intervention. Because of the small sample, the degree to which this group is representative for all donors and donor candidates is unclear. The motivation for participation in the ICBT intervention was high in the group which followed the intervention. Therefore, in future research, the ICBT intervention should be evaluated in a larger sample of kidney donors and donor candidates. Furthermore, the current study merely focused on the subjective evaluation of the intervention to examine its feasibility before applying it to a larger donor population. In the next step, it would be useful to demonstrate the effectiveness of the intervention in comparison with usual psychosocial care for kidney donors and donor candidates, assessing preintervention and postintervention outcomes prospectively. ${ }^{26}$

Kidney donors and donor candidates with mild to moderate levels of distress were invited to follow the ICBT intervention. Some donors indicated that they experienced only minor difficulties, and were therefore not interested in following the intervention. In future research, valid cut-off criteria to screen for psychological distress have to be examined to formulate evidence-based inclusion criteria. In the current study, $21 \%$ of donors or donor candidates who completed the screening tool had a risk profile and participated in the feasibility study. Although this percentage may be considered not to be very high, it is actually rather high if you consider the fact that these donors generally show above-average health ratings before donation. Trying to help each donor who shows physical or emotional limitations is thus relevant, especially also in light of their willingness to undergo surgery to help a patient with end-stage renal disease without direct advantages for themselves and the fact that they often have been or will remain caretakers of a kidney patient. Also, to participate in ICBT interventions, 
general computer skills as well as some knowledge on the use of internet are necessary.

In conclusion, the new ICBT intervention was developed from the donors' perspective based on the results of focus groups and was evaluated positively, both in terms of feasibility (procedure, content, user-friendliness) and perceived effectiveness. Future research should examine the added value of the intervention and the possibility to integrate it into the current psychosocial care for kidney donors.

Acknowledgements The authors would like to thank all donors and donor candidates who participated in the study and the following colleagues for their contributions in the performance of the study: Desiree Pilzecker, Heinrich Kloke, Ine Dooper, Gerben van den Bosch, Simon van Duin, Tessa de Jong-Pulskens and Janneke Vervelde.

Contributors LW: participated in research design, coordination/logistics, performance of the research, data analysis, writing of the paper and approval of the article. HvM and AWME: participated in research design, coordination/logistics, data analysis, revising the paper and approval of the article. CWH: participated in coordination/logistics, acquisition of data, revising the paper and approval of the article. TEB: participated in acquisition of data, focus group interviews, coaching participants, revising the paper and approval of the article. SPFH: participated in acquisition of data, focus group interviews, revising the paper and approval of the article. KAMlvdP: participated in acquisition of data, revising the paper and approval of the article. AJH and LBH: participated in research design, coordination/logistics, revising the paper and approval of the article.

Funding This work was supported by the Dutch Kidney Foundation.

Competing interests AWME, HvM and LW received research grants from the Dutch Kidney Foundation. LBH reports personal fees from Novartis and Chiesi (scientific advisory board). All other authors have no support from any organisation for the submitted work; no financial relationships with any organisations that might have an interest in the submitted work in the previous 3 years; and no other relationships or activities that could appear to have influenced the submitted work.

Patient consent Obtained.

Ethics approval The medical ethics committee of the Radboudumc Nijmegen approved the study (NL.50145.091.14).

Provenance and peer review Not commissioned; externally peer reviewed.

Data sharing statement No additional data available.

Open access This is an open access article distributed in accordance with the Creative Commons Attribution Non Commercial (CC BY-NC 4.0) license, which permits others to distribute, remix, adapt, build upon this work non-commercially, and license their derivative works on different terms, provided the original work is properly cited and the use is non-commercial. See: http://creativecommons.org/ licenses/by-nc/4.0/

(c) Article author(s) (or their employer(s) unless otherwise stated in the text of the article) 2018. All rights reserved. No commercial use is permitted unless otherwise expressly granted.

\section{REFERENCES}

1. Port FK, Wolfe RA, Mauger EA, et al. Comparison of survival probabilities for dialysis patients vs cadaveric renal transplant recipients. JAMA 1993;270:1339-43.

2. Schnuelle P, Lorenz D, Trede M, et al. Impact of renal cadaveric transplantation on survival in end-stage renal failure: evidence for reduced mortality risk compared with hemodialysis during long-term follow-up. J Am Soc Nephrol 1998;9:2135-41.

3. Tonelli M, Wiebe N, Knoll G, et al. Systematic review: kidney transplantation compared with dialysis in clinically relevant outcomes. Am J Transplant 2011;11:2093-109.

4. Wirken $\mathrm{L}$, van Middendorp $\mathrm{H}$, Hooghof $\mathrm{CW}$, et al. The course and predictors of health-related quality of life in living kidney donors: a systematic review and meta-analysis. Am J Transplant 2015;15:3041-54.
5. Gross CR, Messersmith EE, Hong BA, et al. Health-related quality of life in kidney donors from the last five decades: results from the RELIVE study. Am J Transplant 2013;13:2924-34.

6. Clemens KK, Thiessen-Philbrook H, Parikh CR, et al. Psychosocial health of living kidney donors: a systematic review. Am J Transplant 2006;6:2965-77.

7. Tong A, Chapman JR, Wong G, et al. The motivations and experiences of living kidney donors: a thematic synthesis. Am J Kidney Dis 2012;60:15-26.

8. Tong A, Chapman JR, Wong G, et al. Screening and follow-up of living kidney donors: a systematic review of clinical practice guidelines. Transplantation 2011;92:1-72.

9. Duerinckx N, Timmerman L, Van Gogh J, et al. Predonation psychosocial evaluation of living kidney and liver donor candidates: a systematic literature review. Transpl Int 2014;27:2-18.

10. Lidobs recommendations Barcelona. 2014

11. Bahler CD, Sundaram CP. Quality of life following laparoscopic livingdonor nephrectomy. JSLS 2013;17:273-8.

12. Smith GC, Trauer T, Kerr PG, et al. Prospective psychosocial monitoring of living kidney donors using the Short Form-36 health survey: results at 12 months. Transplantation 2004;78:1384-9.

13. Dew MA, DiMartini AF, DeVito Dabbs AJ, et al. Preventive intervention for living donor psychosocial outcomes: feasibility and efficacy in a randomized controlled trial. Am J Transplant 2013;13:2672-84.

14. Jacobs CL, Gross CR, Messersmith EE, et al. Emotional and financial experiences of kidney donors over the past 50 years: the relive study. Clin J Am Soc Nephrol 2015;10:2221-31.

15. Matas AJ, Hays RE, Ibrahim HN. Long-Term Non-End-Stage Renal Disease Risks After Living Kidney Donation. Am J Transplant 2017;17:893-900.

16. Butler AC, Chapman JE, Forman EM, et al. The empirical status of cognitive-behavioral therapy: a review of meta-analyses. Clin Psychol Rev 2006;26:17-31.

17. Hofmann SG, Asnaani A, Vonk IJ, et al. The efficacy of cognitive behavioral therapy: a review of meta-analyses. Cognit Ther Res 2012;36:427-40

18. Andersson G, Cuijpers P, Carlbring P, et al. Guided Internet-based vs. face-to-face cognitive behavior therapy for psychiatric and somatic disorders: a systematic review and meta-analysis. World Psychiatry 2014;13:288-95.

19. Cuijpers $P$, van Straten A, Andersson G. Internet-administered cognitive behavior therapy for health problems: a systematic review. J Behav Med 2008;31:169-77.

20. van Beugen S, Ferwerda M, Hoeve D, et al. Internet-based cognitive behavioral therapy for patients with chronic somatic conditions: a meta-analytic review. J Med Internet Res 2014;16:e88.

21. Johansson R, Andersson G. Internet-based psychological treatments for depression. Expert Rev Neurother 2012;12:861-70.

22. Andersson $\mathrm{G}$. The promise and pitfalls of the internet for cognitive behavioral therapy. BMC Med 2010;8:82.

23. Newman MG, Szkodny LE, Llera SJ, et al. A review of technologyassisted self-help and minimal contact therapies for anxiety and depression: is human contact necessary for therapeutic efficacy? Clin Psychol Rev 2011;31:89-103.

24. Johansson R, Sjöberg E, Sjögren M, et al. Tailored vs. standardized internet-based cognitive behavior therapy for depression and comorbid symptoms: a randomized controlled trial. PLoS One 2012; $7:$ e36905

25. Turk DC. Customizing treatment for chronic pain patients: who, what, and why. Clin J Pain 1990;6:255-70.

26. van Beugen S, Ferwerda M, Spillekom-van Koulil S, et al. Tailored therapist-guided internet-based cognitive behavioral treatment for psoriasis: a randomized controlled trial. Psychother Psychosom 2016;85:297-307.

27. Ferwerda $M$, van Beugen $S$, van Burik $A$, et al. What patients think about E-health: patients' perspective on internet-based cognitive behavioral treatment for patients with rheumatoid arthritis and psoriasis. Clin Rheumatol 2013;32:869-73.

28. Ferwerda $\mathrm{M}$, van Beugen $\mathrm{S}$, van Middendorp $\mathrm{H}$, et al. A tailoredguided internet-based cognitive-behavioral intervention for patients with rheumatoid arthritis as an adjunct to standard rheumatological care: results of a randomized controlled trial. Pain 2017;158:868-78.

29. Hays RD, Morales LS. The RAND-36 measure of health-related quality of life. Ann Med 2001;33:350-7.

30. Zigmond AS, Snaith RP. The hospital anxiety and depression scale. Acta Psychiatr Scand 1983;67:361-70.

31. Vercoulen JH, Swanink CM, Fennis JF, et al. Dimensional assessment of chronic fatigue syndrome. J Psychosom Res 1994;38:383-92.

32. Costa PT, McCrae RR. Domains and facets: hierarchical personality assessment using the revised NEO personality inventory. J Pers Assess 1995;64:21-50. 
33. Boyce P, Parker G. Development of a scale to measure interpersonal sensitivity. Aust N Z J Psychiatry 1989;23:341-51.

34. Dam-Baggen R. De Inventarisatielijst Sociale Betrokkenheid (ISB): een zelfbeoordelingslijst om sociale steun te meten (Inventory for social reliance (ISR): a self-report inventory for the measurement of social support). Gedragstherapie 1992;25:27-46.

35. Busseri MA, Tyler JD. Interchangeability of the Working Alliance Inventory and Working Alliance Inventory, Short Form. Psychol Assess 2003;15:193-7.
36. Horvath AO, Greenberg LS. Development and validation of the Working Alliance Inventory. J Couns Psychol 1989;36:223-33.

37. Ferwerda M, van Beugen S, van Riel PC, et al. Measuring the therapeutic relationship in internet-based interventions. Psychother Psychosom 2016;85:47-9.

38. Elo $\mathrm{S}$, Kyngäs $\mathrm{H}$. The qualitative content analysis process. J Adv Nurs 2008:62:107-15.

39. Cohen J. Statistical power analysis for the behavioral sciences. London UK: Routledge, 1988 